rehabilitation provider: the Accident Compensation Corporation (ACC). Alongside primary self-reported data, electronic hospital discharge data and ACC's compensation and rehabilitation data has been collected.

Results Interviews were completed by $81 \%$ and $79 \%$ of the cohort at the 12 and 24 month time points respectively. Compensation and rehabilitation data has been used to: 1) obtain data for intervening time periods between participant interviews to examine re-injury; 2) provide additional data on compensation exposures, outcomes and costs; 3 ) validate self-reported work-related cause of injury; and 4) to classify injury on the basis of multiple injury diagnoses. Examples of each use will be provided.

Conclusions Linking compensation and rehabilitation data with self-reported survey data has proven valuable by allowing researchers further insight into injury, compensation and rehabilitation factors associated with vocational, functional, and disability outcomes.

\section{DIAGNOSED OCCUPATIONAL CONTACT DERMATITIS COMPARED TO OCCUPATIONAL DERMATITIS WORKERS' COMPENSATION CLAIMS: ARE FEMALES LESS LIKELY TO CLAIM WORKERS' COMPENSATION?}

${ }^{1} G$ Keegel, 'MacFarlane, ${ }^{1}$ Black, ${ }^{2}$ Cahill, 2 Palmer, ${ }^{3}$ Driscoll, ${ }^{2}$ Nixon, ${ }^{1}$ Smith. 'Monash University, Melbourne, Australia; ${ }^{2}$ Occupational Dermatitis Research and Education Centre, Skin \& Cancer Foundation, Melbourne, Australia; ${ }^{3}$ Sydney School of Public Health, The University of Sydney, Sydney, Australia

\subsection{6/oemed-2013-101717.198}

Objective To compare diagnosed disease data for occupational contact dermatitis (OCD) with workers' compensation claims data for OCD, thereby characterising potential differences between the two information sources.

Methods We conducted a retrospective analysis of OCD diagnosed disease and workers' compensation claims data, for the twelve highest risk occupational groups for the state of Victoria, Australia from 1993-2009. Diagnosed disease data for OCD is collected by the Victorian Skin and Cancer Foundation, which services the state of Victoria. Workers' compensation claims for OCD are from the Compensation Research Database, which is held by the Institute for Safety, Compensation and Recovery Research. Estimates of denominators for the occupational groups are derived from 2001 Australian Bureau of Statistics census data. Results The proportions for diagnosed disease and the workers' compensation claims datasets varied considerably by gender, age and occupational group. There was a much higher rate of females diagnosed with OCD compared to the rate of workers' compensation claims (48\% for diagnosed disease dataset vs 33\% for workers' compensation claims dataset, $\mathrm{p}<0.001$ ). These differences by gender were significant in the following occupational groups using diagnosed disease data compared to workers' compensation claims data: Hair and beauty, Automobile workers, Science workers, Trades persons and labourers, Food handlers, and Process workers and packers $(\mathrm{p}<0.05)$.

Conclusions Females were less likely to claim workers' compensation when compared to males even in occupational groups with a predominantly female workforce such as Hair and beauty. The gender discrepancy between the diagnosed disease dataset and the workers' compensation dataset requires further investigation as to why males were more likely to claim than females, particularly as the gender differences for diagnosed disease do not seem to follow the same pattern. A comprehensive surveillance system for OCD policy and practice intervention, should integrate diagnosed disease data and workers' compensation claims statistics.

\section{THE PROMISE AND CHALLENGE OF USING WORKERS COMPENSATION DATA IN COMPARATIVE, CROSS- JURISDICTIONAL RESEARCH}

${ }^{1} \mathrm{C}$ B M Mcleod, ${ }^{2}$ Collie. 'The University of British Columbia, Vancouver, Canada; ${ }^{2}$ Monash University, Melbourne, Australia

\subsection{6/oemed-2013-101717.199}

Comparative, cross-jurisdictional research has the potential to increase our understanding of the causes of work injury and disease and identification of effective prevention and return-towork policies. Results from comparative research may be more powerful than those from single jurisdiction studies as we are able to control for additional sources of variation that may be driving the results and take advantage of policy and program variation across jurisdictions to identify natural experiments that enable stronger causal inference to be drawn. Single jurisdiction studies attempt to do this using variation over time, but often temporal variation is confounded by other factors or is too small for there to be meaningful inference. While the comparative research approach can be powerful, careful attention needs to be paid to the development of comparable study populations and measures and to understanding the differences and/or comparability of the policies under study. Sources of variations across jurisdictions that could lead to spurious differences in the policy outcome need to be identified and controlled for. Fundamentally this implies the need to study the same population at risk, ensuring that cohorts, measures and outcomes are comparable, as well as accounting for other structural or contextual factors that could affect outcomes across jurisdictions. This synthetic presentation will exemplify these challenges with reference to claims and injury data drawn from the Canadian province of British Columbia and the Australian state of Victoria and in comparing claim rates and return-to-work outcomes in cohorts of workers employed in similar occupations and industries. The presentation concludes with a brief overview of a nascent international collaboration that has the aim of creating a network of researchers and data to conduct comparative, cross-jurisdictional occupational health and safety and workers' compensation research in jurisdictions from Oceania, North America and Europe.

\section{Session: Mini symposium IV: Disease surveillance}

\section{THE CONTRIBUTION OF SURVEILLANCE DATA TO OCCUPATIONAL BURDEN OF DISEASE STUDIES}

R Driscoll. University of Sydney, Australia

10.1136/oemed-2013-101717.200

Objectives To explore methodological issues related to the use of surveillance data in burden of disease studies.

Methods and Results Burden of disease methodologies generally rely on the calculation of population attributable fraction (PAF). This in turn typically requires estimates of exposure prevalence and relative risk. An exposure surveillance system can provide the required estimate of exposure prevalence. 
Alternatively, PAF can be estimated if the incidence of the disease in the population, and the incidence of the disease in the unexposed, are known. Improved disease surveillance can make an important contribution in areas where exposure prevalence measures are not good and difficult to improve. Good estimates of disease that can reliably be connected to work (e.g. occupational asthma, occupational dermatitis) in specific study populations would allow the estimate of incidence in the unexposed if the incidence in the population was known. This approach would be more difficult in circumstances where the direct connexion to work is harder to establish (e.g. many musculoskeletal disorders and noise-induced hearing loss). Alternatively, since estimates of the incidence of the disease in the general population will often be available, probably the key area of additional information required would actually be the incidence in unexposed persons. This isn't the typical focus of occupational disease surveillance but could provide a useful contribution to the understanding of occupational disease and its burden on the community.

Thirdly, surveillance systems that cover disorders that are virtually uniquely occupational, such as a register of cases of malignant mesothelioma, can provide a direct estimate of the number of cases of a particular occupational disorder.

Conclusions Occupational surveillance systems can potentially contribute to estimations of burden of disease studies using several different approaches.

\section{HOW USING GEOGRAPHICAL INFORMATION SYSTEMS (GIS) COULD ALLOW US TO IMPROVE OCCUPATIONAL DISEASES (OD) SURVEILLANCE?}

${ }^{1} \mathrm{M}$ D Delaunay, ${ }^{2}$ Godard, ${ }^{3}$ Bicout, ${ }^{4}$ de Gaudemaris, ${ }^{4}$ Bonneterre. ${ }^{1}$ Paris, France; ${ }^{2}$ UMR CNRS 3258, Maison des sciences de l'homme (MSH), Paris Nord, Saint-Denis, La Plaine, France; ${ }^{3}$ UJF-Grenoble 1/CNRS/TIMC-IMAG UMR 5525 (EPSP team), Grenoble, France; ${ }^{4}$ Grenoble Teaching Hospital : Occupational and Environmental Diseases Centre, Grenoble, France

\subsection{6/oemed-2013-101717.201}

Objective To show and illustrate to what extent, GIS could be an opportunity for work-related and occupational diseases surveillance, in combining relevant "spatialisable" information from different data sources, in order to help analyse and present existing data in a systematic, and easily understandable way; which might be of great help for surveillance, vigilance, and prevention purposes.

Methods Cases of Work related diseases ("numerator") from different and complementary sources, as well as data related to underlying industrial tissue and working population according to activity sectors ("denominator") are to be georeferenced and projected (mapped together) with GIS software. For the purpose of our illustration, numerator data are those from the French National Surveillance Scheme on OD Surveillance and Prevention rnv3p, and data from a compensated scheme; data from the denominator are files of enterprises from Chambers of Commerce and Industry as well as from the French National Health insurance company for salaried workers.

Supplementary qualitative knowledge might also be gathered, georeferenced and mapped to increase the level of information produced (here: data from "on the ground" by occupational physicians). We used ArcGIS ( $9^{\text {th }}$ and $10^{\text {th }}$ version) software and basemaps from the National Geographic Institute (IGN) and OpenStreetMap (OSM).

Results Results are illustrated by maps derived from analyses concerning one specific activity sector. The differences and complementarities in case capture between the work-related diseases surveillance scheme and the compensated OD register are highlighted. The spreading of some toxicological risks from companies to their subcontractors is also shown.

Conclusion This methodology, by combining different data sources and a convenient visualisation of the results, is of a great help to adopt a systemic and integrate point of view on OD. Furthermore, it might help surveillance systems to better analyse some issues of concern (capture, shading zone, geographical patterns of referrals or reporting, etc[3DOTS]).

Acknowledgements rnv3p, ANSES, CNAM-TS.

\section{INFORMING PUBLIC POLICY IN OCCUPATIONAL HEALTH THROUGH DISEASE SURVEILLANCE DATA - THE 'THOR' EXAMPLE. (MINISYMPOSIUM: OCCUPATIONAL DISEASE SURVEILLANCE)}

R M Agius. University of Manchester, Manchester, United Kingdom

10.1136/oemed-2013-101717.202

Objectives To discuss how voluntary occupational health surveillance can help informing public policy in relation to health at work.

Methods and Results The Health and Occupation Research Network (THOR) collects incidence data on occupational disease and work related ill health (WRI).

Incidence data from THOR has been used to inform and direct UK policy and priorities in relation to occupational asthma and dermatitis such as in bakers and spray painters. THOR played an important part in measuring the UK's "Revitalising Health and Safety" targets: It was the main UK data source used to report the statistically significant decrease in both work-related asthma and skin disease in the decade after 2009. The effects of interventions, such as implementation of statute, relating to chromate, latex, and glutaraldehyde were studied using incidence data and demonstrated the value of these public policy initiatives. 'Before and after' comparisons were made to investigate changes in sickness absence certification following the introduction of the 2010 'fitnote' which assisted GPs to recommend workplace adjustments. In the first year the new category certified as 'fit' with recommended adjustments was almost exclusively limited to that proportion of workers previously certified as 'fit'. However after the first year there was evidence that the GPs were also making rehabilitation recommendations in those who would previously have been certified as 'sick'.

A collaborative international consortium entitled MODERNET (Monitoring trends in Occupational Diseases and tracing new and Emerging Risks in a Network) has been established with EU funding. MODERNET is developing improvements in data quality, trends analysis and disease vigilance, intended to influence public policy at a supra-national level.

Conclusions Data from voluntary occupational health surveillance schemes are valuable in informing public policy for example by monitoring trends in WRI incidence, and in evaluating the benefits of interventions.

203

INTERVENTIONS FOR REDUCING THE UNDERREPORTING OF OCCUPATIONAL DISEASES - MINISYMPOSIUM 'IMPROVING THE IMPACT OF OCCUPATIONAL DISEASE SURVEILLANCE' 